\title{
BMJ Open Usefulness of natriuretic peptide for the diagnosis of Kawasaki disease: a systematic review and meta-analysis
}

\author{
Kuan-Ho Lin, ${ }^{1,2}$ Shy-Shin Chang, ${ }^{3,4}$ Chin-Wei Yu, ${ }^{5,6}$ Shen-Che Lin,, \\ Shu-Chun Liu, ${ }^{5,7}$ Hsiao-yun Chao, ${ }^{5,7}$ Meng-tse Gabriel Lee, ${ }^{8}$ Jiunn-Yih Wu, ${ }^{5,6}$ \\ Chien-Chang Lee $8,9,10$
}

To cite: Lin K-H, Chang S-S, Yu C-W, et al. Usefulness of natriuretic peptide

for the diagnosis of Kawasaki disease: a systematic review and meta-analysis. BMJ Open 2015;5: 006703 .

doi:10.1136/bmjopen-2014006703

- Prepublication history for this paper is available online. To view these files please visit the journal online (http://dx.doi.org/10.1136/ bmjopen-2014-006703).

Received 22 September 2014 Revised 13 March 2015 Accepted 18 March 2015

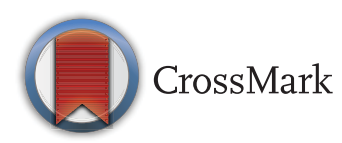

For numbered affiliations see end of article.

Correspondence to

Dr Jiunn-Yih Wu;

asepsis.wu@msa.hinet.net and

Dr Chien-Chang Lee; cclee100@gmail.com

\section{ABSTRACT}

Objective: To examine the diagnostic value of serum B-type natriuretic peptide (BNP) in acute Kawasaki disease (KD).

Design: Systematic review and meta-analysis.

Data sources: A systematic literature search strategy was designed and carried out using MEDLINE, EMBASE and the Cochrane Library from inception to December 2013. We also performed manual screening of the bibliographies of primary studies and review articles, and contacted authors for additional data.

Study eligibility criteria: We included all BNP and NT-pro (N-terminal prohormone) BNP assay studies that compared paediatric patients with KD to patients with febrile illness unrelated to KD. We excluded case reports, case series, review articles, editorials, congress abstracts, clinical guidelines and all studies that compared healthy controls.

Primary and secondary outcome measures: The performance characteristics of BNP were summarised using forest plots, hierarchical summary receiver operating characteristic (ROC) curves and bivariate random effects models.

Results: We found six eligible studies including 279 cases of patients with KD and 203 febrile controls. Six studies examined NT-proBNP and one examined BNP. In general, NT-proBNP is a specific and moderately sensitive test for identifying KD. The pooled sensitivity was $0.89(95 \% \mathrm{Cl} 0.78$ to 0.95$)$ and the pooled specificity was $0.72(95 \% \mathrm{Cl} 0.58$ to 0.82$)$. The area under the summary ROC curve was $0.87(95 \% \mathrm{Cl}$ 0.83 to 0.89 ). The positive likelihood ratio ( $L R+3.20$, $95 \% \mathrm{Cl} 2.10$ to 4.80 ) was sufficiently high to be qualified as a rule-in diagnostic tool in the context of high pre-test probability and compatible clinical symptoms. A high degree of heterogeneity was found using the Cochran $Q$ statistic.

Conclusions: Current evidence suggests that NT-proBNP may be used as a diagnostic tool for KD. NT-proBNP has high diagnostic value for identifying $K D$ in patients with protracted undifferentiated febrile illness. Prospective large cohort studies are needed to help determine best cut-off values and further clarify the role of NT-proBNP in the diagnosis process of $\mathrm{KD}$.

\section{Strengths and limitations of this study}

- Currently, diagnosis of Kawasaki disease (KD) in the early course of disease remains challenging, and our review suggests that N-terminal prohormone serum B-type natriuretic peptide (NT-proBNP) may represent a valuable diagnostic tool for KD.

- A bivariate random effect model was used to account for the inherent negative correlation arising from different cut-off values reported by different studies.

- Summary likelihood ratios were used to measure predictive accuracy.

- Considerable heterogeneity was found in the pooled estimates and, unfortunately, the small number of studies does not allow for further exploration of the source of heterogeneity by sensitivity analysis or meta-regression.

- There are limited data available for analysis of $K D$ diagnostic accuracy at different cut-off values, so we cannot conclusively suggest the best cut-off value.

\section{INTRODUCTION}

Kawasaki disease (KD) is a potentially fatal acute childhood vasculitis. It is the most common cause of acquired heart disease in children in developed countries. ${ }^{1}$ The morbidity and mortality associated with $\mathrm{KD}$ is primarily caused by coronary artery aneurysms, which may occur in $20-25 \%$ of untreated patients. $^{12}$ Early treatment with intravenous immunoglobulin and, recently, with the addition of prednisolone, has been shown to reduce the incidence of coronary aneurysms. On the contrary, if the treatment is delayed, the incidence of coronary artery aneurysms may dramatically increase. ${ }^{2}$

Timely diagnosis of $\mathrm{KD}$ is sometimes a challenging task for clinicians. There is no specific test or pathognomonic clinical features for definitive diagnosis of $\mathrm{KD}$. The 
epidemiological case definition includes fever and at least four of five clinical criteria: changes in the extremities, polymorphous exanthema, bilateral conjunctival injection, changes in the lips and oral cavity, and cervical adenopathy. ${ }^{1}{ }^{3}$ Sometimes, patients have some major clinical features but do not fulfil the complete diagnostic criteria. These patients are diagnosed as having 'incomplete' or 'atypical' KD. They are more commonly young infants and carry the same risk for coronary artery aneurysms as those with complete presentations. ${ }^{4}$ Failure to diagnose these patients is associated with increased coronary morbidity. Echocardiography usually plays a key role in early diagnosis of children with incomplete $\mathrm{KD}$, but it requires a specialised technique and is not widely available for frontline physicians. A biomarker that can indicate early on the possibility of cardiac involvement in patients with protracted fever may help select appropriate patients for further confirmatory cardiac sonography.

B-type natriuretic peptide (BNP) or its inactive cleavage product N-terminal pro-BNP (NT-proBNP) is synthesised and released by ventricular cardiomyocytes in response to myocardial wall stress and ischaemia. ${ }^{5}$ Elevated levels of BNP and NT pro-BNP have been shown to be associated with adverse outcomes in a number of settings, including acute coronary syndrome, congestive heart failure and patients undergoing major cardiac surgery. ${ }^{6}$ Myocardial involvement in $\mathrm{KD}$ is widely recognised. The cardiac pathology associated with KD includes coronary artery aneurysms, myocarditis, endocarditis, mild valvular regurgitation and pericardial effusion. ${ }^{7-9}$

Recently, a number of studies have examined the predictive value of BNP or NT-proBNP for differentiating KD from other febrile illnesses. ${ }^{3}{ }^{10-17}$ The accuracy estimates of individual studies, however, vary widely among studies. We therefore carried out a systemic review and meta-analysis to summarise evidence on the usefulness of this biomarker in the assessment of patients in whom $\mathrm{KD}$ was suspected.

\section{METHODS}

We followed the PRISMA guidelines for meta-analysis of diagnostic studies in our data extraction, analysis and reporting. ${ }^{18}$

\section{Study selection}

We searched three electronic databases (PubMed, EMBASE and the Cochrane Library), through December 2013, for records of studies that evaluated the diagnostic performance of serum or plasma BNP for diagnosis of KD. We used broad search terms to avoid missing studies. The search terms used were "natriuretic peptide or b-type natriuretic peptide or n-terminal pro-b type natriuretic peptide or BNP or NT-proBNP" crossed with 'Kawasaki disease or mucocutaneous lymph node syndrome'. The same strategy with EMTREE tools was used to search the EMBASE database without setting any time or language restrictions. We also performed manual screening of the bibliographies of primary studies and review articles and contacted authors for additional data. Initial eligibility was determined independently by two reviewers. Disagreements between the reviewers were resolved by additional reviewers in a consensus meeting.

We included studies comparing patients with $\mathrm{KD}$ and patients with febrile illness unrelated to KD. For inclusion, studies were required to have a study population of paediatric patients. Studies that compared healthy controls were not eligible for inclusion, because they tend to overestimate the sensitivity and specificity. BNP assays from any manufacturer were eligible. We excluded case reports, case series, review articles, editorials, congress abstracts and clinical guidelines. The study inclusion and exclusion process is summarised in figure 1.

\section{Data extraction}

From each of the included studies, we extracted information about the publication (title, authors and journal), study population, comparison group, study design (case-control or cohort), assay used for BNP or NT-proBNP measurement, cut-off value, criteria used for the diagnosis of $\mathrm{KD}$ and data for $2 \times 2$ contingency tables. We also extracted patient data regarding the sensitivity and specificity with the BNP or NT-proBNP assay. If multiple cut-offs were reported in one study, we consistently used the cut-off that optimised the Youden index.

\section{Quality assessment of the included studies}

Methodological quality was assessed using the quality assessment for studies of diagnostic accuracy (QUADAS) tool. ${ }^{19}$ We used the international diagnostic criteria for $\mathrm{KD}$ as a reference standard. The spectrum of patients included in a study was considered to be representative of the target population if the patients had clinical manifestation suspected of $\mathrm{KD}$. We considered partial and differential verification bias if all the paediatric patients were not assessed with the same reference standard. In addition, we considered there was no incorporation bias if the diagnosis of $\mathrm{KD}$ was established strictly based on the KD diagnostic criteria, regardless of the value of serum BNP or NT-proBNP levels.

\section{Data analysis}

To evaluate the diagnostic performance of BNP testing for $\mathrm{KD}$, we performed a comparison evaluating the performance of BNP assays for case patients with proven KD compared with control febrile patients without KD. Data were extracted to construct $2 \times 2$ tables, which were used to calculate sensitivity and specificity.

The sensitivity and specificity estimates were pooled by using bivariate random effects regression models based on the recommendation of the Cochrane Diagnostic Test Accuracy Working Group. The bivariate model takes into consideration the potential negative correlation between sensitivity and specificity by explicitly incorporating this correlation factor as well as between-study 
Figure 1 Flow chart describing systematic literature search and study selection process.

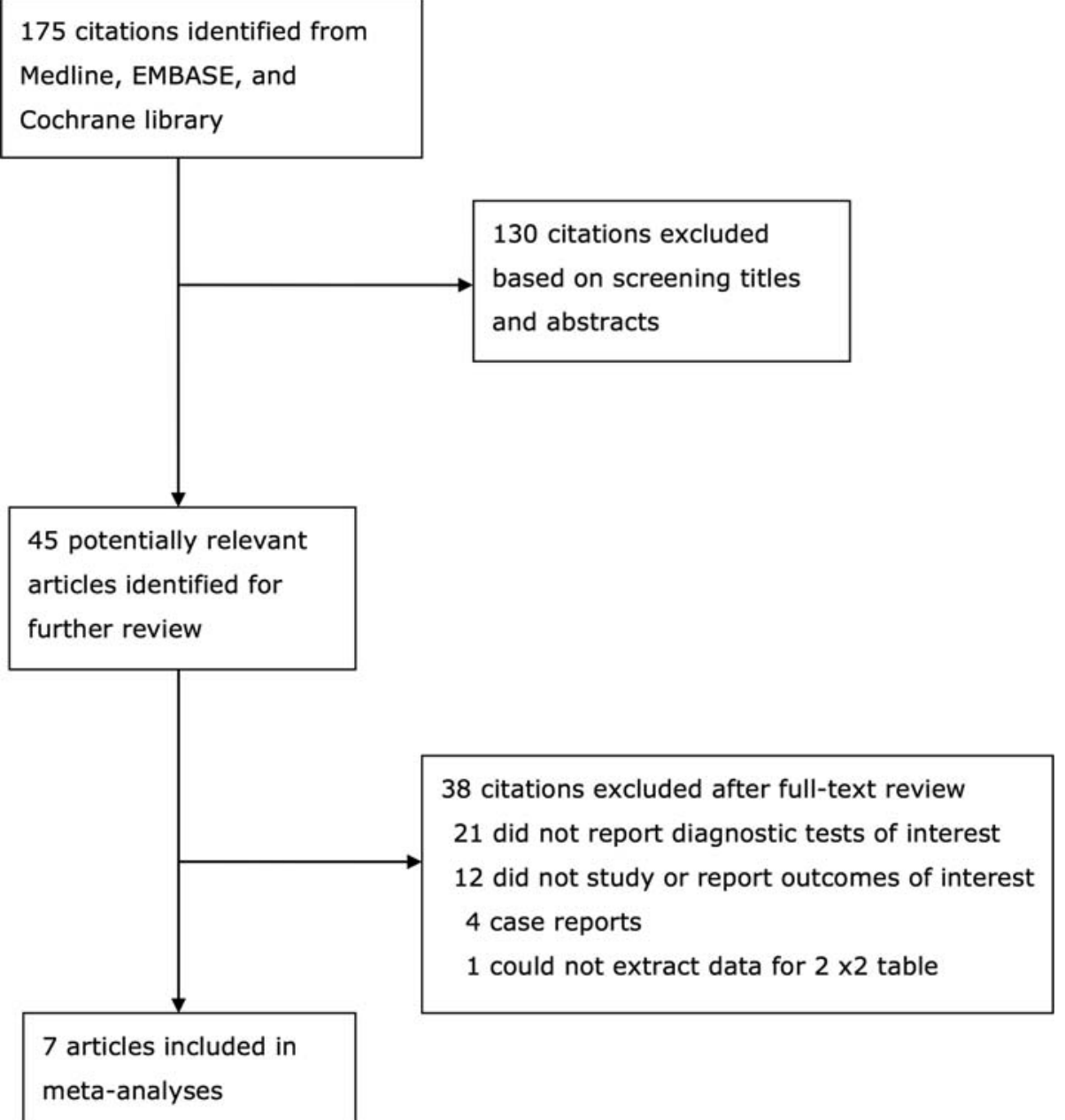

heterogeneity in the analysis. ${ }^{19}$ The model was also used to construct a hierarchical summary receiver operating characteristic (HSROC) curve and to calculate the area under the curve (AUC). Confidence regions for the summary points and the prediction regions of $95 \%$ of future studies were plotted in the HSROC. To deal with values of zero in $2 \times 2$ contingency tables, continuity correction was performed by adding half to each cell. This correction also helped to reduce the small study bias. Heterogeneity between studies was tested using the Cochran $Q$ statistic $(\mathrm{p}<0.05)$ and quantified with the $\mathrm{I}^{2}$ statistic. The value of the $\mathrm{I}^{2}$ statistic describes the variation of effect size that is attributable to heterogeneity across studies. ${ }^{20}$ Statistically significant heterogeneity was considered present if $\mathrm{I}^{2}$ was greater than $50 \%$. Subgroup analyses were performed on studies using the NT-proBNP or BNP assays. We tested the publication bias by Egger's test for the asymmetry of funnel plots by regression methods. Skewed and asymmetrical funnel plots indicate the presence of publication bias. ${ }^{21}$ All statistical analyses were conducted using the STATA software V.11.0 (Stata Corp, College Station, Texas, USA). All statistical tests were two tailed, and statistical significance was defined as a $\mathrm{p}$ value less than 0.05 .

\section{RESULTS}

In total, 175 studies (excluding duplicates) were identified using the search strategy outlined above (figure 1). After the first round of screening of title and abstracts, 131 ineligible studies, case reports, or reviews were excluded. Forty-four potentially relevant studies were retrieved for full text evaluation, of which 38 studies were further excluded for varying reasons, leaving 6 that met the inclusion criteria.

The seven eligible studies included 428 patients of confirmed KD and 709 control febrile patients without KD. Table 1 presents a summary of the characteristics of the seven included studies and patients. The number of patients with $\mathrm{KD}$ in each study ranged from 6 to 149, and their mean/median ages ranged from 19 to 52 months. All studies used the appropriate prospective case-control designs and the outcome is verified by the classical clinical diagnostic criteria for $\mathrm{KD}$, which requires the presence of 5 days and four of the five principal clinical features. ${ }^{2}$ Six studies measured the NT-proBNP at the acute stage of $\mathrm{KD}$, while only one study measured BNP. The cut-off values of NT-proBNP ranged from 98 to $260 \mathrm{pg} / \mathrm{mL}$. Table 2 summarised the characteristics of the test kit, characteristics of control 
Table 1 Characteristics of patients for included studies

\begin{tabular}{lcllll}
\hline Author, country, year & $\begin{array}{l}\text { Case/ } \\
\text { controls (N) }\end{array}$ & $\begin{array}{l}\text { Mean age } \\
\text { (months) }\end{array}$ & $\begin{array}{l}\text { Biomarkers } \\
\text { tested }\end{array}$ & $\begin{array}{l}\text { Sensitivity, } \\
\text { Cut-off }\end{array}$ & $\begin{array}{l}\text { specificity } \\
\text { Kawamura T, Japan, 2000 }\end{array}$ \\
\hline Lee H, Korea, 2006 & $52 / 26(58)$ & 21 & BNP & $16.8 \mathrm{pg} / \mathrm{mL}$ & $0.97,0.86$ \\
Zhang QY, China, 2006 & $6 / 16(22)$ & 19 & NT-proBNP & $260 \mathrm{ng} / \mathrm{L}$ & $0.93,0.88$ \\
Dahdah N, Canada, 2009 & $43 / 19(62)$ & 47.1 & NT-proBNP & $103.6 \mathrm{ng} / \mathrm{L}$ & 1,1 \\
Cho SY, Korea, 2011 & $59 / 59(108)$ & 33.2 & NT-proBNP & $235.2 \mathrm{ng} / \mathrm{L}$ & $0.66,0.77$ \\
McNeal-Davidson A, Canada, 2012 & $81 / 49(130)$ & 42 & NT-proBNP & $190 \mathrm{ng} / \mathrm{L}$ & $0.89,0.69$ \\
Shiraishi M, Japan, 2013 & $149 / 506(655)$ & 33 & NT-proBNP & $98 \mathrm{pg} / \mathrm{mL}$ & $0.98,0.47$ \\
\hline
\end{tabular}

BNP, B-type natriuretic peptide; NT-proBNP, N-terminal prohormone serum BNP.

patients and mean serum levels of NT-proBNP or BNP in case or control patients. All studies used age matched patients with febrile illness unrelated to $\mathrm{KD}$ as the controls. Six studies reported NT-proBNP levels, and three studies reported BNP levels. Mean serum levels of NT-proBNP were between 750 and $1511 \mathrm{pg} / \mathrm{mL}$ in patients with $\mathrm{KD}$, in contrast to 47 and $199 \mathrm{pg} / \mathrm{mL}$ in the control group. Mean serum levels of BNP were between 52 and $142 \mathrm{pg} / \mathrm{mL}$ in patients with $\mathrm{KD}$, in contrast to 4 and $60 \mathrm{pg} / \mathrm{mL}$ in the control group.

We used the QUADAS tool for study quality assessment. Figure 2 provides an overall impression of the methodological quality of the studies. All blood drawn was taken in proximity to the confirmation diagnosis. All patients were verified by the same reference standards in all studies. None of the included studies explained the withdrawals or uninterested results. Only two of six studies reported the physicians were blinded to the index test while verifying outcomes by reference standards. We could not exclude the possibility of incorporation bias.

\section{Diagnostic accuracy indices}

Results of the meta-analysis indicated that PCT testing has an acceptable accuracy regarding differentiating $\mathrm{KD}$ from other causes of prolonged febrile illness (table 3). The pooled sensitivity and specificity estimates were 0.89 (95\% CI 0.79 to 0.95 ) and 0.77 (95\% CI 0.62 to 0.88 ), respectively. When the analysis was restricted to six studies measuring NT-proBNP, there is no change in sensitivity $(0.89,95 \%$ CI 0.78 to 0.95$)$ but a slight decrease in specificity $(0.72,95 \%$ CI 0.58 to 0.82$)$. The positive likelihood ratio (LR+, 3.20, 95\% CI 2.10 to 4.80$)$ of NT-proBNP is not sufficient for a standalone rule-in test. The negative likelihood ratio (LR-, $0.15,95 \%$ CI 0.07 to $0.31)$, in the context of low pretest probability $(<10 \%)$, could reduce the post-test probability to such a level that $\mathrm{KD}$ could be safely excluded. To take within and between study variation into account, we constructed HSROC and forest plot for NT-proBNP, which derived an area under the curve (AUC) of 0.87 (95\% CI 0.83 to 0.89 ) and a summary OR of 21.6 (95\% CI 8.33 to 55.97 ), respectively (figures 3 and 4). According to Hosmer and Lemeshow, our AUC of between 0.80 and 0.90 can be regarded as 'good'. ${ }^{22}$ High statistical heterogeneity was also noted, but the Galbraith plots did not indicate a major outlier, which, for the most part, could account for the observed heterogeneity. ${ }^{23}$ Results of Eggers tests are presented in table 3 , but the small number of studies may prevent a meaningful exam of publication bias.

\section{DISCUSSION}

We present a systematic review assessing the diagnostic accuracy of serum BNP or NT-proBNP measurement in the clinical setting of KD. We found that NT-proBNP can be a useful marker for the diagnosis of KD. The plasma NT-proBNP level was elevated in patients with $\mathrm{KD}$ in the acute phase, whereas it was only mildly elevated in febrile control patients. Unfortunately, according to the rules of thumb by Jaeschke in interpreting sizes of likelihood ratios, our positive likelihood ratio for NT-proBNP provides only weak evidence for KD diagnosis. ${ }^{24}$ Even though NT-proBNP is not sufficient for a standalone rule-in test, the widely available blood test may help clinicians decide whether there is a need to arrange confirmatory cardiac sonographic examination for suspected patients. Cardiac sonographic examination is rarely available in front-end settings such as emergency departments or in ambulatory clinics. In addition, the current standard for diagnosis of KD is largely based on empirical clinical criteria, and it will be interesting for others to find out whether incorporating NT-proBNP test results would further enhance the accuracy of current criteria in diagnosing atypical KD.

In addition, we calculated the post-test probability to make our results more informative to clinicians. In a group of patients with a $20 \%$ pretest probability of $\mathrm{KD}$, a positive NT-proBNP test with a LR+ of 3.20 would increase the post-test probability to $44 \%$, and a negative NT-proBNP test with a LR- of 0.15 would reduce the post-test probability to $4 \% .^{25}$ Therefore, NT-proBNP measurements had a moderate rule-in value and a good rule-out value for the diagnosis of $\mathrm{KD}$ among febrile patients. We cannot draw any conclusions on the comparative diagnostic value between BNP and NT-proBNP tests because only one study reported data on BNP.

Currently, diagnosis of $\mathrm{KD}$ in the early course of disease remains challenging. Classical clinical diagnostic 


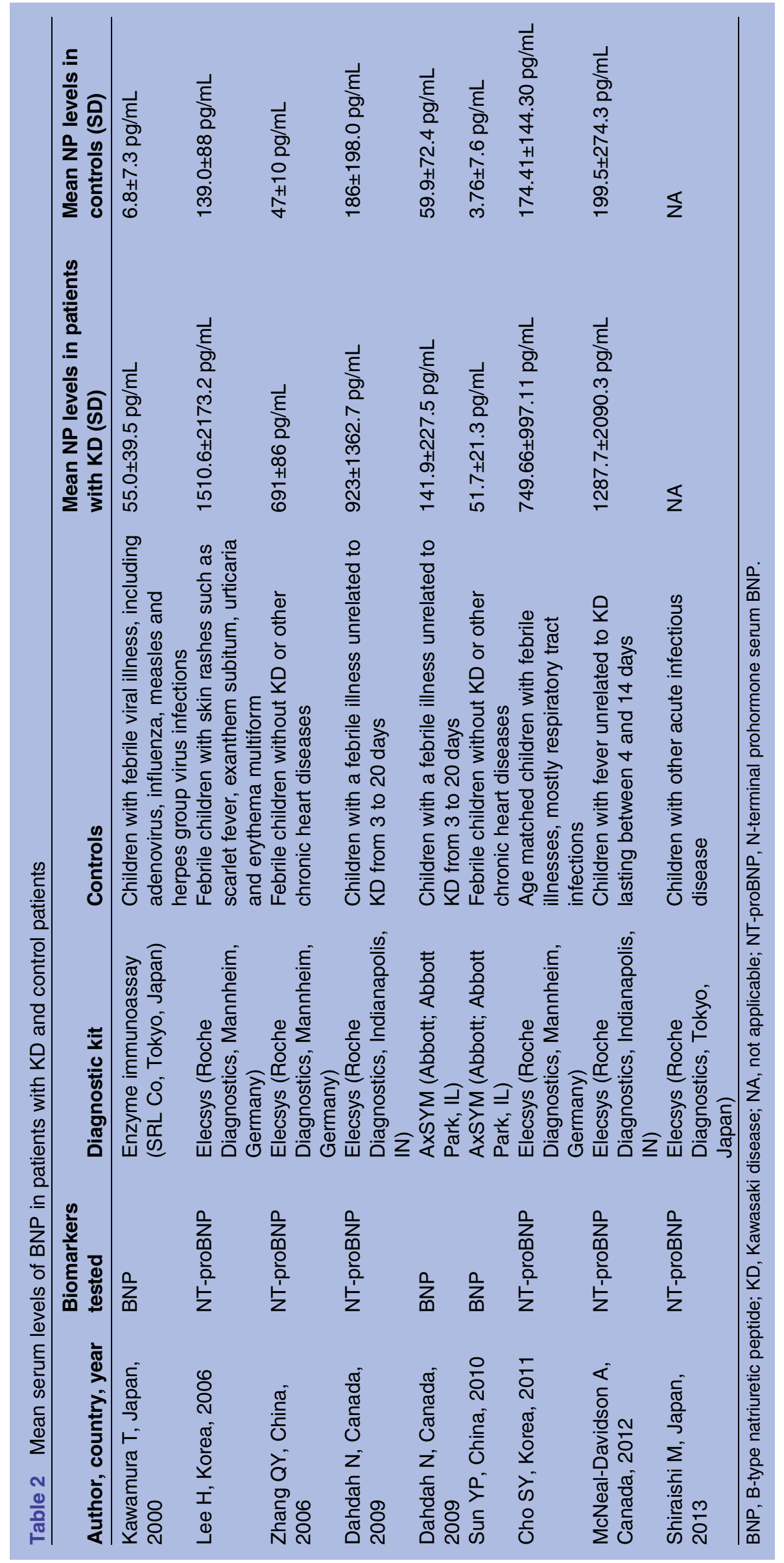




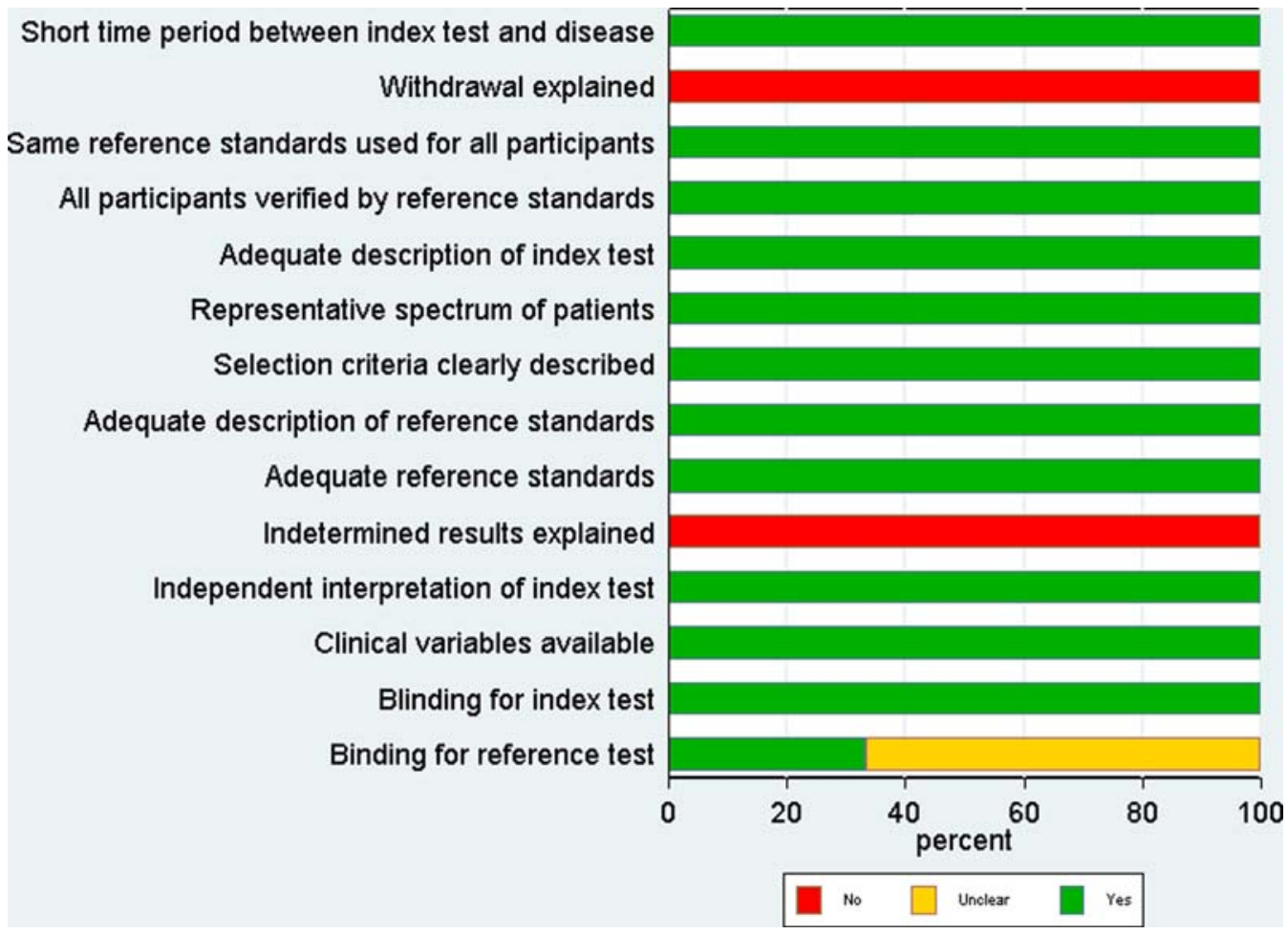

Figure 2 Quality of included studies.

criteria are sufficient to exclude KD. Some laboratory findings have shown their usefulness in identifying $\mathrm{KD}$ : the presence of raised $\mathrm{C}$ reactive protein (CRP), a raised white cell count, urine analysis showing white cell counts under microscopy, and raised transaminases or bilirubin. ${ }^{26}$ However, these tests are neither pathognomonic nor diagnostic. Without a valid biomarker for predicting $\mathrm{CA}$ aneurysms in patients with $\mathrm{KD}$, Harada et al and Beiser et al developed instruments that incorporate age, gender, leucocyte count, fever duration, haemoglobin levels and CRP levels, to predict CA aneurysms. ${ }^{27}$ The accuracy of these instruments was not consistently high in several validation studies. For patients with incomplete clinical manifestations, transthoracic echocardiography is usually required. This diagnostic procedure is expensive and has limited availability at different settings. Therefore, until now, diagnosis and the timesensitive immunoglobulin therapy are usually delayed until a fever has persisted for at least 5 days. Our study showed NT-proBNP measurement may represent a major advance in the diagnostic pathways in patients with suspected KD.

Serum BNP is elevated due to ventricular wall stress imposed by volume or pressure overload. In addition to the haemodynamic stress, inflammation of the myocardial tissue may also induce the production of BNP. ${ }^{28}$ Immunohistochemical analysis of endomyocardial biopsy specimens from patients with myocarditis showed immunoreactivity for BNP and histological change such as myocyte necrosis, inflammatory infiltrates and fibrosis were more prominent in immunohistochemically positive biopsy specimens. ${ }^{28}$ It has been shown that the cardiac pathology, including endocardium, myocardium and pericardium can be found in nearly all patients with KD. ${ }^{79}$ The vasculitis and myocarditis associated with $\mathrm{KD}$ may thus be an important cause of BNP elevation, even in the absence of functional haemodynamic change. Limited by the studies available for BNP testing, we cannot draw any conclusion regarding the accuracy of BNP in comparison with NT-proBNP. In adult reports, BNP and NT-proBNP are equally accurate in diagnosing acute heart failure. For clinical use, NT-proBNP may have some advantages over BNP. NT-proBNP has a longer half-life (60-120 min) than BNP (20-30 min) and the interpretation of serum levels of NT-proBNP is less affected by kidney functions. ${ }^{5}$ Another advantage of NT-proBNP is its stability at room temperature.

The interpretation of the NT-proBNP levels in paediatric patients is complicated because children have the physiological secretion of BNP in early life. According to previous work, the upper limit for age (95th centile) is $646 \mathrm{ng} / \mathrm{L}$ for infants between 1 month and 1 year, $413 \mathrm{ng} / \mathrm{L}$ for children between 1 and 2 years, $289 \mathrm{ng} / \mathrm{L}$ for those between 2 and 6 years, and $157 \mathrm{ng} / \mathrm{L}$ for those $>6$ years. ${ }^{29}$ We do not recommend these cut-off levels for differentiating between $\mathrm{KD}$ and other febrile illness. The mean age of patients for the included studies ranged from 19 to 47 months, and the best cut-off values recommended by each study varied between 103 and $260 \mathrm{ng} / \mathrm{L}$, depending on the characteristics of febrile illness in the comparison group. Based on the two studies with the largest sample sizes, a cut-off value 


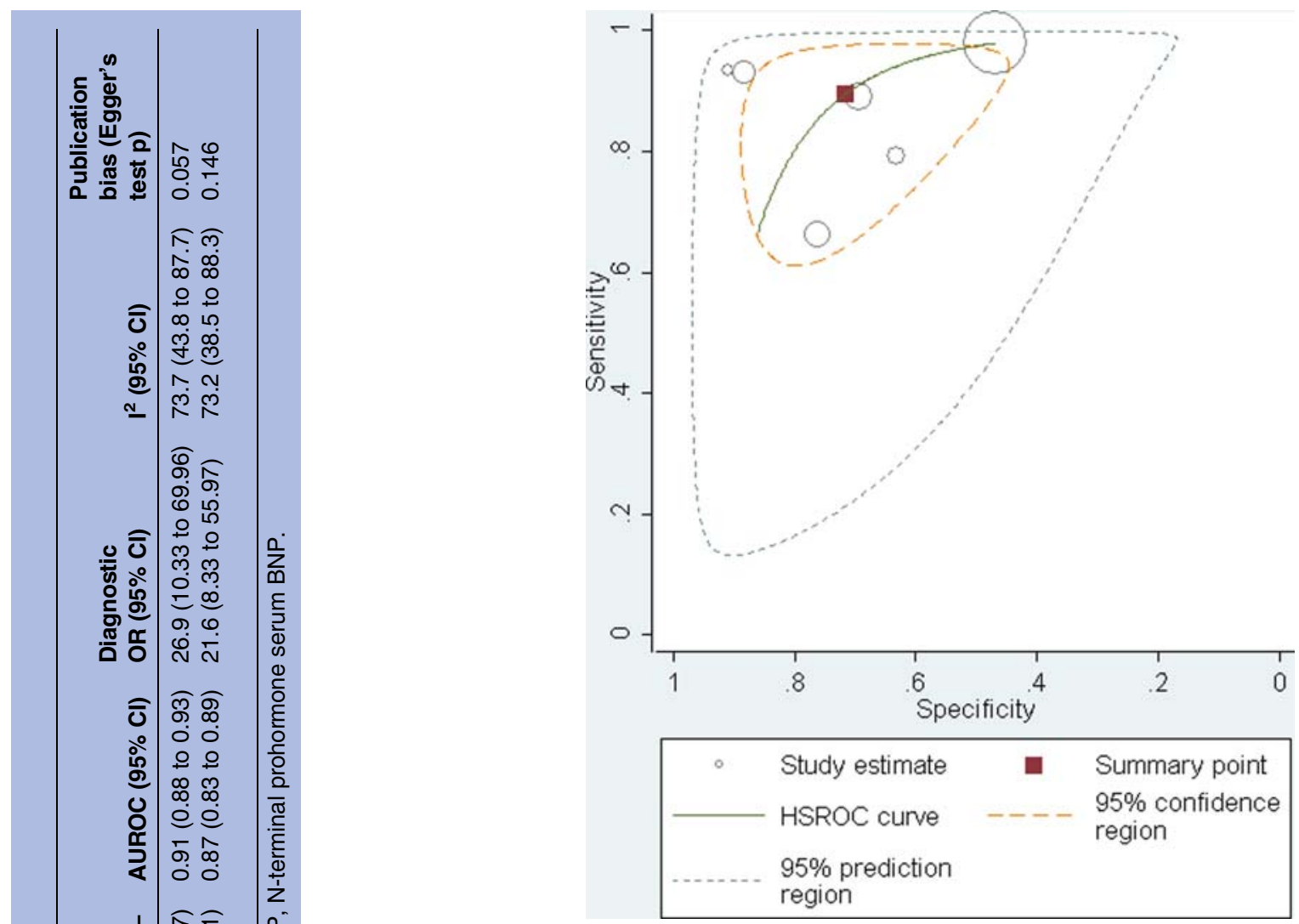

Figure 3 Hierarchical summary receiver-operator curves (HSROC) for NT-proBNP (NT-proBNP, N-terminal prohormone serum B-type natriuretic peptide).

between 190 and $260 \mathrm{ng} / \mathrm{L}$ may be a recommended cut-off value for this meta-analysis.

\section{Limitation}

The results of this study have to be interpreted in the context of several limitations. First, considerable heterogeneity was found in the pooled estimates. The small number of studies does not allow further exploration of the source of heterogeneity by sensitivity analysis or meta-regression. Some factors that may have contributed to this heterogeneity cannot be assessed or adjusted. For example, duration of fever and symptoms of the case patients and the characteristics and severity of febrile illness in the control group are likely to have an important effect on BNP testing performance. These factors are not reported in detail and their effect cannot be assessed. Second, the case patients enrolled in the studies include patients with $\mathrm{KD}$ with several types of cardiac pathologies, including those without CA aneurysms. Results of this study did not provide support for NT-proBNP to be used as an important diagnostic tool for the medical decision of intravenous immunoglobulin (IVIG) therapy. Third, given the limited data available for analysis of KD diagnostic accuracy at different cut-off values, no strong conclusions for the best BNP cut-off value use can be reached without further large cohort studies. The ideal approach for determining the cut-off 


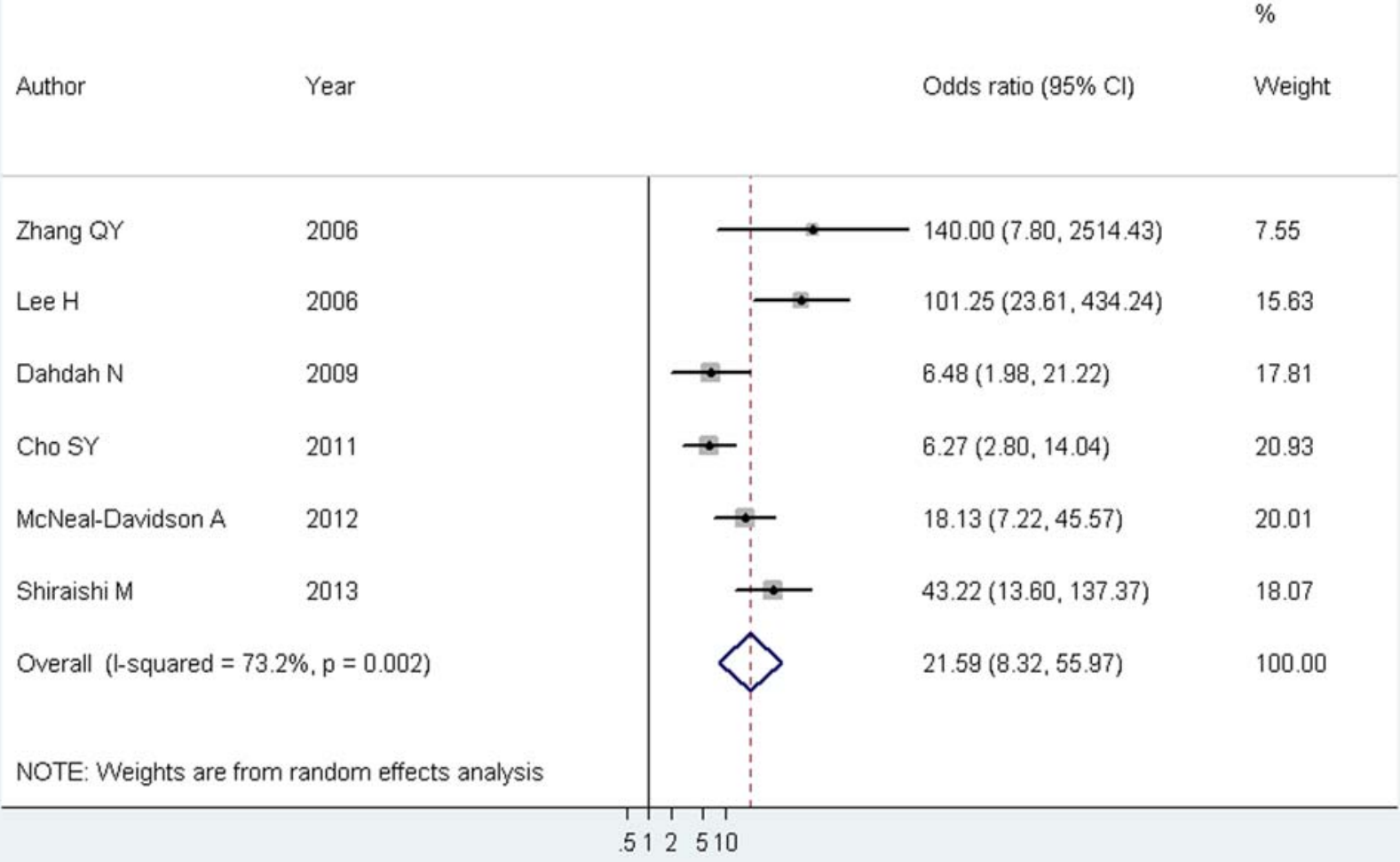

Figure 4 Forest plot showing the diagnostic value of serum B-type natriuretic peptide in acute Kawasaki disease (KD) in different studies.

value for NT-proBNP or BNP testing in the diagnosis of $\mathrm{KD}$ would be to carry out a prospective, cohort study in consecutive patients with clinical suspicion of $\mathrm{KD}$. Before this type of study is available, individual data meta-analysis may also provide better insight into the age-specific best cut-off value in this clinical setting.

\section{CONCLUSIONS}

To date, KD is diagnosed on clinical grounds alone. Our review shows determination of BNP in clinical routine may represent a valuable addition to the current diagnostic work up of patients with suspected KD. The measurement of this biomarker is likely to help screen out some patients with incomplete $\mathrm{KD}$ presentations who may need cardiac imaging studies for early identification of possible cardiac complications. Considering the small sample size and suboptimal case-control design of the currently available studies, a sufficiently powered prospective cohort study is needed to conclusively address the usefulness of serum NT-proBNP as a diagnostic aid in $\mathrm{KD}$.

\section{Author affiliations}

${ }^{1}$ College of Medicine, China Medical University, Taichung, Taiwan

${ }^{2}$ Department of Emergency Medicine, China Medical University Hospital,

Taichung, Taiwan
${ }^{3}$ Department of Family Medicine, Chang Gung Memorial Hospital, Taoyuan, Taiwan

${ }^{4}$ Graduate Institute of Clinical Medical Sciences, College of Medicine, Chang Gung University, Taoyuan, Taiwan

${ }^{5}$ Chang Gung University College of Medicine, Taoyuan, Taiwan

${ }^{6}$ Department of Emergency Medicine, Chang Gung Memorial Hospital, Keelung, Taiwan

${ }^{7}$ Department of Emergency Medicine, Chang Gung Memorial Hospital, Taoyuan, Taiwan

${ }^{8}$ Department of Emergency Medicine, National Taiwan University Hospital, Taipei, Taiwan

${ }^{9}$ Department of Emergency Medicine, National Taiwan University Hospital Yunlin Branch, Douliou, Taiwan

${ }^{10}$ Department of Epidemiology, Harvard School of Public Health, Boston, USA

Acknowledgements The authors thank the staff of the Core Labs, Department of Medical Research, National Taiwan University Hospital, for technical support.

Contributors $\mathrm{K}-\mathrm{HL}$ was involved in data management, statistics, first draft, final draft and approval; S-SC in scientific and statistics advice, study monitoring and final draft; C-WY in scientific advice, and first and final draft; S-CLin in scientific advice, and final draft; S-CLiu in statistics advice and final draft; H-YC in final draft; M-TGL in final draft; J-YW in statistics, scientific and statistic advice, first draft, final draft and approval; C-CL in design, scientific and statistics advice, first draft, final draft and approval.

Funding The study was supported by National Taiwan University Hospital Yunlin Branch Research Grant, NTUHYL102S008.

Competing interests None declared.

Provenance and peer review Not commissioned; externally peer reviewed. 
Data sharing statement The study results will be published in a peer-reviewed scientific journal. The extracted data will be open to sharing on request.

Open Access This is an Open Access article distributed in accordance with the Creative Commons Attribution Non Commercial (CC BY-NC 4.0) license, which permits others to distribute, remix, adapt, build upon this work noncommercially, and license their derivative works on different terms, provided the original work is properly cited and the use is non-commercial. See: http:// creativecommons.org/licenses/by-nc/4.0/

\section{REFERENCES}

1. Burns JC, Mason WH, Glode MP, et al. Clinical and epidemiologic characteristics of patients referred for evaluation of possible Kawasaki disease. United States Multicenter Kawasaki Disease Study Group. J Pediatr 1991;118:680-6.

2. Newburger JW, Takahashi M, Gerber MA, et al. Diagnosis, treatment, and long-term management of Kawasaki disease: a statement for health professionals from the Committee on Rheumatic Fever, Endocarditis and Kawasaki Disease, Council on Cardiovascular Disease in the Young, American Heart Association. Circulation 2004;110:2747-71.

3. Kishimoto S, Suda K, Teramachi Y, et al. Increased plasma type B natriuretic peptide in the acute phase of Kawasaki disease. Pediatr Int 2011;53:736-41.

4. Sonobe T, Kiyosawa N, Tsuchiya K, et al. Prevalence of coronary artery abnormality in incomplete Kawasaki disease. Pediatr Int 2007;49:421-6.

5. Nakao K, Ogawa Y, Suga S, et al. Molecular biology and biochemistry of the natriuretic peptide system. II: natriuretic peptide receptors. J Hypertens 1992;10:1111-14.

6. de Lemos JA, McGuire DK, Drazner MH. B-type natriuretic peptide in cardiovascular disease. Lancet 2003;362:316-22.

7. Fujiwara H, Hamashima Y. Pathology of the heart in Kawasaki disease. Pediatrics 1978;61:100-7.

8. Yutani C, Go S, Kamiya T, et al. Cardiac biopsy of Kawasaki disease. Arch Pathol Lab Med 1981;105:470-3.

9. Yonesaka S, Takahashi T, Matubara T, et al. Histopathological study on Kawasaki disease with special reference to the relation between the myocardial sequelae and regional wall motion abnormalities of the left ventricle. Jpn Circ J 1992;56:352-8.

10. McNeal-Davidson A, Fournier A, Spigelblatt L, et al. Value of amino-terminal pro B-natriuretic peptide in diagnosing Kawasaki disease. Pediatr Int 2012;54:627-33.

11. Bang S, Yu JJ, Han MK, et al. Log-transformed plasma level of brain natriuretic peptide during the acute phase of Kawasaki disease is quantitatively associated with myocardial dysfunction. Korean $J$ Pediatr 2011;54:340-4.
12. Cho SY, Kim Y, Cha SH, et al. Adjuvant laboratory marker of Kawasaki disease; NT-pro-BNP or hs-CRP? Ann Clin Lab Sci 2011;41:360-3.

13. Dahdah N, Siles A, Fournier A, et al. Natriuretic peptide as an adjunctive diagnostic test in the acute phase of Kawasaki disease. Pediatr Cardiol 2009;30:810-17.

14. Kawamura $\mathrm{T}$, Wago $\mathrm{M}$, Kawaguchi $\mathrm{H}$, et al. Plasma brain natriuretic peptide concentrations in patients with Kawasaki disease. Pediatr Int 2000;42:241-8.

15. Kurotobi S, Kawakami N, Shimizu K, et al. Brain natriuretic peptide as a hormonal marker of ventricular diastolic dysfunction in children with Kawasaki disease. Pediatr Cardiol 2005;26:425-30.

16. Sun YP, Wang WD, Zheng XC, et al. [Levels of serum brain natriuretic peptide and the correlation to heart function in children with Kawasaki disease]. Zhongguo Dang Dai Er Ke Za Zhi 2010;12:169-71.

17. Shiraishi M, Fuse $\mathrm{S}$, Mori T, et al. N-terminal pro-brain natriuretic peptide as a useful diagnostic marker of acute Kawasaki disease in children. Circ J 2013;77:2097-101.

18. Leeflang MM, Deeks JJ, Gatsonis C, et al. Systematic reviews of diagnostic test accuracy. Ann Intern Med 2008;149:889-97.

19. Whiting $P$, Rutjes AW, Reitsma JB, et al. The development of QUADAS: a tool for the quality assessment of studies of diagnostic accuracy included in systematic reviews. BMC Med Res Methodol 2003;3:25.

20. Higgins JP, Thompson SG, Deeks JJ, et al. Measuring inconsistency in meta-analyses. BMJ 2003;327:557-60.

21. Egger M, Davey Smith G, Schneider M, et al. Bias in meta-analysis detected by a simple, graphical test. BMJ 1997;315:629-34.

22. Hosmer DWaL, S. Applied logistic regression. New York: John Wiley \& Sons, Inc, 2000.

23. Galbraith RF. A note on graphical presentation of estimated odds ratios from several clinical trials. Stat Med 1988;7:889-94.

24. Jaeschke R, Guyatt GH, Sackett DL, et al. Users' guides to the medical literature: III. How to use an article about a diagnostic test $\mathrm{B}$. What are the results and will they help me in caring for my patients? JAMA 1994;271:703-7.

25. Akobeng AK. Understanding diagnostic tests 2: likelihood ratios, pre- and post-test probabilities and their use in clinical practice. Acta Paediatr 2007;96:487-91.

26. Nakamura $\mathrm{Y}$, Yashiro M, Uehara R, et al. Use of laboratory data to identify risk factors of giant coronary aneurysms due to Kawasaki disease. Pediatr Int 2004;46:33-8.

27. Zhao LL, Wang YB, Suo L. [Meta-analysis of the risk factors for coronary artery lesion secondary to Kawasaki disease in Chinese children]. Zhonghua Er Ke Za Zhi 2011;49:459-67.

28. Takemura G, Fujiwara $\mathrm{H}$, Takatsu $\mathrm{Y}$, et al. Venticular expression of atrial and brain natriuretic peptides in patients with myocarditis. Int $J$ Cardiol 1995;52:213-22.

29. Nir A, Lindinger A, Rauh M, et al. NT-pro-B-type natriuretic peptide in infants and children: reference values based on combined data from four studies. Pediatr Cardiol 2009;30:3-8. 